\title{
Singular Reduction of Poisson Manifolds
}

\author{
JUAN-PABLO ORTEGA ${ }^{1 \star}$ and TUDOR S. RATIU ${ }^{2 \star \star}$ \\ ${ }^{1}$ Département de Mathématiques, École Polytechnique Fédérale de Lausanne, CH 1015 Lausanne, \\ Switzerland.e-mail: Juan-Pablo.Ortega@epfl.ch \\ ${ }^{2}$ Department of Mathematics, University of California, Santa Cruz, Santa Cruz, CA 95064, USA, \\ and Département de Mathématiques, École Polytechnique Fédérale de Lausanne, CH 1015 \\ Lausanne, Switzerland.e-mail: Tudor.Ratiu@epfl.ch
}

(Received: 18 September 1998)

\begin{abstract}
The conditions under which it is possible to reduce a Poisson manifold via a regular foliation have been completely characterized by Marsden and Ratiu. In this Letter we show that this characterization can be generalized in a natural way to the singular case and, as a corollary, we obtain that when the singular distribution is given by the tangent spaces to the orbits created by a Hamiltonian Lie group action, one reproduces the Universal Reduction Procedure of Arms, Cushman, and Gotay.
\end{abstract}

Mathematics Subject Classifications (1991): 70H33, 58F05, 53C80.

Key words: Poisson manifold, reduction, symmetry, singularity, stratification.

\section{Introduction}

Reduction theory is the standard method within the framework of Hamiltonian dynamics for taking advantage of the conserved quantities associated with the symmetries of a problem. See [1, 14], and references therein for an exposition of this subject.

The symmetries of a system are usually expressed in terms of a Hamiltonian Lie group action. However, as it was already known to É. Cartan [9], the natural mathematical objects that one should look at when carrying out reduction are foliations. When one takes as the foliation the tangent spaces to the orbits created by a free Hamiltonian Lie group action, one recovers the group-theoretical approach to the symmetries of the system. This degree of generality has proven to be extremely convenient when formulating necessary and sufficient conditions for the reducibility of a problem in the Poisson category [11].

\footnotetext{
^ Research partially supported by a Fulbright/BCH Fellowship, the US Information Agency and a Rotary Ambassadorial Scholarship.

$\star \star$ Research partially supported by NSF Grant DMS-9802378, DOE Contract DE-FG0395ER25245-A000, and FNS Grant 21-54138.98.
} 
The goal of this Letter is to show a generalization of the reducibility characterization in [11], to the case in which the distribution used for the reduction process presents singularities. The results obtained will allow us to reproduce in a straightforward manner some standard results in the theory of singular reduction by Hamiltonian Lie group actions.

\section{Stratified Poisson Reduction by Foliations}

We first introduce some concepts that will be used in the exposition.

DEFINITION 2.1. Let $M$ be a differentiable manifold. A collection of subspaces $D_{m} \subset T_{m} M$ is called a smooth or differentiable distribution if there are locally defined smooth vector fields $\left\{X_{i}\right\}_{i \in I}$ in $\mathfrak{X}(M)$, such that $\left\{X_{i}(m)\right\}_{i \in I}$ spans $D_{m}$.

(i) $D$ is called integrable if for any $m \in M$, there is an injectively immersed submanifold $S_{m} \subset M$, such that $T_{m} S_{m}=D_{m}$.

(ii) $D$ is called involutive if it is invariant under the (local) flows associated to vector fields with values in $D$.

Remark 2.2. The definition of involutive distribution given above is more general than the traditional one, that is, the Lie bracket $[X, Y]$ takes values in $D$ whenever $X$ and $Y$ are vector fields with values in $D$. The two concepts of involutivity are equivalent only when the dimension of $D_{m}$ is independent of $m \in M$.

THEOREM 2.3 (Generalized Frobenius Theorem). A differentiable distribution D on a manifold $M$ is integrable iff it is involutive.

Proof. See [10, 12, 16, 17].

DEFINITION 2.4. Let $M$ be a differentiable manifold and $S \subset M$ be a subset of $M$. We say that $S$ is a stratified subset of $M$ with strata $\left\{S_{i}\right\}_{i \in I}$ when

(S1) The subsets $S_{i} \subset S, i \in I$, are injectively immersed submanifolds of $M$ and form a partition of $S$.

(S2) The partition of $S$ into the connected components $\left\{S_{i}^{j}\right\}_{i \in I}^{j \in J}$ of the subsets $S_{i}$ is locally finite.

(S3) If $S_{i}^{j} \cap \operatorname{cl}\left(S_{i^{\prime}}^{j^{\prime}}\right) \neq \varnothing$ for $(i, j) \neq\left(i^{\prime}, j^{\prime}\right)$, then $S_{i}^{j} \subset S_{i^{\prime}}^{j^{\prime}}$ and $\operatorname{dim}\left(S_{i}^{j}\right)<$ $\operatorname{dim}\left(S_{i^{\prime}}^{j^{\prime}}\right)$.

(S4) $\operatorname{cl}\left(S_{i}\right) \backslash S_{i}$ is a disjoint union of strata of dimension strictly less than $\operatorname{dim}\left(S_{i}\right)$.

We define the tangent bundle $T S$ of the stratified subset $S$ as $T S=\bigcup_{i \in I} T S_{i}$, where $T S_{i}$ denote the ordinary tangent bundles of the manifolds $S_{i}$.

DEFINITION 2.5. Let $M$ be a differentiable manifold and $S \subset M$ be a stratified subset of $M$ with strata $\left\{S_{i}\right\}_{i \in I}$. We say that $\left.D \subset T M\right|_{S}$ is a smooth distribution on $S$ adapted to the stratification $\left\{S_{i}\right\}_{i \in I}$, if $D \cap T S_{i}$ is a smooth distribution on $S_{i}$ for 
all $i \in I$. The distribution $D$ is said to be integrable if $D \cap T S_{i}$ is integrable for each $i \in I$.

In the situation described by the previous definition, the integrability of the distributions $D \cap T S_{i}$ on $S_{i}$ allows us to partition each $S_{i}$ into the maximal integral manifolds. Thus, there is an equivalence relation $\Phi_{i}$ on $S_{i}$ whose equivalence classes are precisely these maximal integral manifolds. Doing this on each $S_{i}$, we obtain an equivalence relation $\Phi$ on the whole set $S$ by taking the union of the different equivalence classes corresponding to all the $\Phi_{i}$. We define the quotient space $S / \Phi$ as $S / \Phi:=\bigcup_{i \in I} S_{i} / \Phi_{i}$.

Notice that Definition 2.4 does not require the stratified subset $S$ to be a smooth manifold. In fact, during part of our discussion we will work with structures somewhat more general than manifolds, namely varieties.

DEFINITION 2.6. A pair $\left(X, C^{\infty}(X)\right)$, where $X$ is a topological space and $C^{\infty}(X)$ $\subset C^{0}(X)$ is a subset of continuous functions on $X$, is called a variety with smooth functions $C^{\infty}(X)$. If $Y \subset X$ is a subset of $X$, the pair $\left(Y, C^{\infty}(Y)\right)$ is said to be a subvariety of $\left(X, C^{\infty}(X)\right)$, if $Y$ is a topological space endowed with the relative topology defined by that of $X$ and

$$
C^{\infty}(Y)=\left\{f \in C^{0}(Y)|f=F|_{Y} \text { for some } F \in C^{\infty}(X)\right\} .
$$

Sometimes $C^{\infty}(Y)$ is called the set of Whitney smooth functions on $Y$ with respect to $X$. A map $\varphi: X \rightarrow Z$ between two varieties is said to be smooth when it is continuous and $\varphi^{*} C^{\infty}(Z) \subset C^{\infty}(X)$.

In our discussion, we will consider $\left(S, C^{\infty}(S)\right)$ as a subvariety of $\left(M, C^{\infty}(M)\right)$. $S / \Phi$ is a variety whose set of smooth functions is defined by the requirement that the canonical projection $\pi: S \rightarrow S / \Phi$ is a smooth map, that is,

$$
\begin{aligned}
C^{\infty}(S / \Phi) & :=\left\{f \in C^{0}(S / \Phi) \mid f \circ \pi \in C^{\infty}(S)\right\} \\
& =\left\{f \in C^{0}(S / \Phi)|f \circ \pi=F|_{S} \text { for some } F \in C^{\infty}(M)\right\} .
\end{aligned}
$$

We will consider the case in which the distribution $D$ is given by the tangent spaces to the orbits of a Lie group $G$ acting smoothly on $M$. By construction, $D$ is integrable (the maximal integral manifolds are the orbits). We will be particularly interested in the case in which the $G$-action is proper. For future reference, we quote the following result in relation with this particular kind of action:

PROPOSITION 2.7. Let $G$ be a Lie group acting properly on the manifold $M$. Let $\left(S, C^{\infty}(S)\right)$ be a subvariety of $\left(M, C^{\infty}(M)\right)$ such that $S$ is a $G$-invariant subset of $M$. Then each $G$-invariant function $f \in C^{\infty}(S)^{G}$ on $S$ can be extended to $M$ in a $G$-invariant fashion, that is, there is a $F \in C^{\infty}(M)^{G}$ such that $\left.F\right|_{S}=f$.

Proof. See [3, Proposition 2]. 
DEFINITION 2.8. Let $(M,\{\cdot, \cdot\})$ be a Poisson manifold and $D \subset T M$ be a smooth distribution on $M$. The distribution $D$ is called Poisson or canonical, if the condition $\left.\mathbf{d} f\right|_{D}=\left.\mathbf{d} g\right|_{D}=0$ for $f, g \in C^{\infty}(M)$ implies that $\left.\mathbf{d}\{f, g\}\right|_{D}=0$

If the distribution $D$ is defined by the tangent spaces to the orbits of a Lie group $G$ acting smoothly on $M$, the condition that $D$ is Poisson can be expressed in the following way: if $f, g \in C^{\infty}(M)$ are such that $\xi_{M}[f]=\xi_{M}[g]=0$ for any $\xi \in \mathfrak{g}$, then $\xi_{M}[\{f, g\}]=0$, for any $\xi \in \mathfrak{g}$, where $\xi_{M}$ denotes the infinitesimal generator of the action.

DEFINITION 2.9. Let $S \subset M$ be a stratified subset, $g \in C^{\infty}(S)$, and $m \in S$. A local extension of $g$ at $m$ is a function $G \in C^{\infty}(M)$ satisfying the following condition: there exists an open neighborhood $U_{m}$ of $m$ in $M$ such that $\left.G\right|_{S \cap U_{m}}=$ $\left.g\right|_{S \cap U_{m}}$.

Let $D$ be an integrable distribution adapted to the stratified subset $S \subset M$. We say that $D$ has the extension property if for any $f \in C^{\infty}(S / \Phi)$ and any $m \in S$ the map $f \circ \pi \in C^{\infty}(S)$ admits a local extension $F \in C^{\infty}(M)$ at $m$ such that $\left.\mathbf{d} F\right|_{D}=0$ (at all points of $M$ ).

Remark 2.10. Note that if $S$ is just a submanifold of $M$ and $D$ has constant dimension, that is, $D$ is a usual smooth integrable subbundle of $T M$, the extension property is satisfied automatically: it suffices to take a submanifold chart of $S$ relative to $M$ which is also a foliated chart of $S$ with respect to the distribution $\left.D\right|_{S}$. Also, if $D$ is given by the tangent spaces to the orbits of a proper $G$-action on $M$ and $S$ is a $G$-invariant subset of $S$, Proposition 2.7 guarantees that the triplet $(M, S, D)$ has the extension property. In general, note that given two different points $m, m^{\prime} \in S$, the local extensions at $m$ and at $m^{\prime}$ need not coincide.

DEFINITION 2.11. Let $(M,\{\cdot, \cdot\})$ be a Poisson manifold, $S$ be a stratified subset of $M$ with strata $\left\{S_{i}\right\}_{i \in I}$, and $\left.D \subset T M\right|_{S}$ be a Poisson integrable distribution adapted to $S$ such that $(M, S, D)$ has the extension property. We say that $(M, S, D)$ is Poisson reducible if the pair $\left(C^{\infty}(S / \Phi),\{\cdot, \cdot\}_{S / \Phi}\right)$ is a well-defined Poisson algebra, where the bracket $\{\cdot, \cdot\}_{S / \Phi}$ is given by

$$
\{f, g\}_{S / \Phi}(\pi(m))=\{F, G\}(m),
$$

for every $m \in S$, where $F, H \in C^{\infty}(M)$ are smooth local extensions of $f \circ \pi, g \circ$ $\pi \in C^{\infty}(S)$ at $m$ satisfying $\left.\mathbf{d} F\right|_{D}=\left.\mathbf{d} G\right|_{D}=0$.

Below we shall use the following notation: if $V$ is a vector space and $W \subset V$ is a subspace, the annihilator $W^{\circ}$ of $W$ in the dual $V^{*}$ of $V$ is defined by

$$
W^{\circ}=\left\{\alpha \in V^{*} \mid \alpha(w)=0 \text {, for all } w \in W\right\} .
$$


We now give a necessary and sufficient condition for $(M, S, D)$ to be Poisson reducible. This result naturally generalizes the result of Marsden and Ratiu [11] to the singular case.

THEOREM 2.12. Let $(M,\{\cdot, \cdot\})$ be a Poisson manifold with Poisson tensor B: $T^{*} M \rightarrow T M, S$ be a stratified subset of $M$ with strata $\left\{S_{i}\right\}_{i \in I}$, and $\left.D \subset T M\right|_{S}$ be a Poisson integrable distribution adapted to $S$ such that $(M, S, D)$ has the extension property. Then $(M, S, D)$ is Poisson reducible if and only if for any $m \in S$ we have

$$
B\left(\Delta_{m}\right) \subset T_{m} S+\left[\Delta_{m}^{S}\right]^{\circ},
$$

where

$$
\Delta_{m}:=\left\{\mathbf{d} F(m)\left|F \in C^{\infty}(M), \mathbf{d} F\right|_{D}=0\right\},
$$

and

$$
\begin{aligned}
\Delta_{m}^{S}:=\left\{\mathbf{d} F(m) \in \Delta_{m} \mid\right. & \left.F\right|_{U_{m} \cap S} \text { is constant, for } U_{m} \text { an open } \\
& \text { neighborhood of } m \text { in } M\} .
\end{aligned}
$$

Proof. If $F \in C^{\infty}(M)$, denote by $X_{F}$ the Hamiltonian vector field defined by $F$. An alternative way to write the condition in the statement is

$$
\begin{aligned}
\left\{X_{F}(m) \mid F \in\right. & \left.C^{\infty}(M),\left.\mathbf{d} F\right|_{D}=0\right\} \\
\subset T_{m} S+ & \left\{\mathbf{d} F(m) \mid F \in C^{\infty}(M),\right. \\
& \left.\mathbf{d} F\right|_{D}=0,\left.F\right|_{U_{m} \cap S} \text { is constant, for } U_{m} \text { an open } \\
& \text { neighborhood of } m \text { in } M\}^{\circ} .
\end{aligned}
$$

The proof of the theorem follows the strategy of [11]. First, we suppose that $(M, S, D)$ is Poisson reducible. Let $F \in C^{\infty}(M)$ satisfy $\left.\mathbf{d} F\right|_{D}=0$ and let

$$
\alpha_{m} \in\left[T_{m} S+\left[\Delta_{m}^{S}\right]^{\circ}\right]^{\circ}=\left[T_{m} S\right]^{\circ} \cap \Delta_{m}^{S} .
$$

Thus, $\alpha_{m}=\mathbf{d} K(m)$ for some $K \in C^{\infty}(M)$ satisfying $\left.\mathbf{d} K\right|_{D}=0, K$ is constant on $U_{m} \cap S$, where $U_{m}$ is an open neighborhood of $m$ in $M$. Therefore, the functions $F$ and $K$ induce functions $f, k \in C^{\infty}(S / \Phi)$ by $f \circ \pi=F \circ i, k \circ \pi=K \circ i$ and $k$ is constant in an open neighborhood of $\pi(m)$ in $S / \Phi$. Thus, by Poisson reducibility (2.1),

$$
\left\langle\alpha_{m}, X_{F}(m)\right\rangle=\{K, F\}(m)=\{k, f\}_{S / \Phi}(\pi(m))=0,
$$

since $k$ is a constant in a neighborhood of $\pi(m)$. Since $\alpha_{m} \in\left[T_{m} S+\left[\Delta_{m}^{S}\right]^{\circ}\right]^{\circ}$ is arbitrary, it follows that $X_{F}(m) \in T_{m} S+\left[\Delta_{m}^{S}\right]^{\circ}$.

Conversely, if $B\left(\Delta_{m}\right) \subset T_{m} S+\left[\Delta_{m}^{S}\right]^{\circ}$, let $f, g \in C^{\infty}(S / \Phi)$ and $F, G \in$ $C^{\infty}(M)$ be smooth local extensions of $f \circ \pi, g \circ \pi \in C^{\infty}(S)$ at $m$ such that $\left.\mathbf{d} F\right|_{D}=\left.\mathbf{d} G\right|_{D}=0$. Since $D$ is a Poisson distribution, it follows that $\left.\mathbf{d}\{F, G\}\right|_{D}=$ 0 , which implies that $\{F, G\}$ is constant on the equivalence classes of $\Phi$ and 
therefore induces a function, which we shall call $\{f, g\}_{S / \Phi} \in C^{\infty}(S / \Phi)$, satisfying the condition (2.1). If we show that this function does not depend on the extensions involved, this defines the reduced bracket $\{f, g\}_{S / \Phi}$ on $S / \Phi$. Indeed, let $G^{\prime} \in C^{\infty}(M)$ be another local extension of $g \circ \pi \in C^{\infty}(S)$ at $m$ such that $\left.\mathbf{d} G^{\prime}\right|_{D}=0$. Then $\left.\left(G-G^{\prime}\right)\right|_{S \cap U_{m}}=0$, where $U_{m}$ is the neighborhood of $m$ in $M$ given by the hypothesis of local extendability of pull backs of functions from the quotient. Thus, $\mathbf{d}\left(G-G^{\prime}\right)(m)$ vanishes on $T_{m} S$. It also vanishes on $\left[\Delta_{m}^{S}\right]^{\circ}$ by definition. Now using the hypothesis, for any $m \in S$

$$
\left\langle\mathbf{d}\left(G-G^{\prime}\right)(m), B(m)(\mathbf{d} F(m))\right\rangle=0, \quad \text { hence } \quad\{F, G\}(m)=\left\{F, G^{\prime}\right\}(m),
$$

which proves the independence on how $g \circ \pi$ is extended. By antisymmetry of $\{\cdot, \cdot\}$ it is also independent of the extension of $f \circ \pi$, therefore $\{f, g\}_{S / \Phi}$ is well-defined and uniquely determined by the expression (2.1). With this bracket $\left(C^{\infty}(S / \Phi)\right.$, $\left.\{\cdot, \cdot\}_{S / \Phi}\right)$ is a Poisson algebra since the bracket $\{\cdot, \cdot\}_{S / \Phi}$ inherits all the properties of a Poisson bracket from those of $\{\cdot, \cdot\}$.

Remark 2.13. In the regular case considered in [11], $S$ is a submanifold and $D$ is a smooth subbundle of $T M$. We have already seen that in this situation the extension property is automatically satisfied. The condition of Poisson reducibility is stated as

$$
B\left(D^{\circ}\right) \subset T S+D .
$$

Since the distribution $D$ is adapted to the submanifold $S$, working in a chart on $M$ around a given point $m \in S$, any $\alpha_{m} \in D_{m}^{\circ}$ can be written as $\mathbf{d} F(m)$ for some smooth function $F$ defined in this chart and constant on the local leaves of the foliation given by $D$. Now choose in every chart some function that is constant on the leaves of the foliation and construct a smooth function on $M$ by adding all these functions by means of a partition of unity. The resulting smooth function, also called $F$, is constant on the leaves of the foliation (since $\left.\mathbf{d} F\right|_{D}=0$ by construction) and has the same differential at $m$, that is, $\mathbf{d} F(m)=\alpha_{m}$. This shows that in the regular case $\Delta_{m}=D_{m}^{\circ}$.

Let us now show that in the regular case, $T_{m} S+D_{m}=T_{m} S+\left[\Delta_{m}^{S}\right]^{\circ}$. Since $\Delta_{m}^{S} \subset$ $\Delta_{m}$, it follows that $D_{m}=\left[\Delta_{m}\right]^{\circ} \subset\left[\Delta_{m}^{S}\right]^{\circ}$ and, hence, $T_{m} S+D_{m} \subset T_{m} S+\left[\Delta_{m}^{S}\right]^{\circ}$. To prove the converse, it suffices to show that

$$
\left[\Delta_{m}^{S}\right]^{\circ} \subset T_{m} S+D_{m}=\left[T_{m} S\right]^{\circ \circ}+\left[\Delta_{m}\right]^{\circ}=\left[\left(T_{m} S\right)^{\circ} \cap \Delta_{m}\right]^{\circ},
$$

or, equivalently, that $\left(T_{m} S\right)^{\circ} \cap \Delta_{m} \subset \Delta_{m}^{S}$ which is proved in the following way. If $\alpha_{m} \in\left(T_{m} S\right)^{\circ} \cap \Delta_{m}$, then $\alpha_{m}=\mathbf{d} F(m)$ for $F \in C^{\infty}(M)$ satisfying $\left.\mathbf{d} F\right|_{D}=$ 0 and $\left.\mathbf{d} F(m)\right|_{T_{m} S}=0$. One can replace $F$ by a smooth function vanishing on the distribution $D$ and at the same time being constant in $U \cap S$, for $U$ an open neighborhood of $m$ in $M$. (To do this, replace in a chart at $m$ the function $\left.F\right|_{U}$ by the constant function equal to $\alpha_{m}$, which is possible since $\alpha_{m}$ vanishes on $D_{m}$ and 
on $T_{m} S$, and then patch this function with the restriction of $F$ to an open set $V$ such that $U \cup V=M$ by means of a partition of unity. The resulting function is smooth, satisfies $\left.\mathbf{d} F\right|_{D}=0$ and is constant on $U \cap S$.) This function has differential at $m$ belonging to $\Delta_{m}^{S}$ which proves the desired inclusion.

It should be also noted that in the singular case condition (2.3) is only sufficient for the Poisson reducibility of $(M, S, D)$, even if $D$ is given by a group action. For example, consider the case of the $S^{1}$ action by positive rotations on the complex line $\mathbb{C}$. We take the integral manifolds of the distribution $D$ to be the concentric circles and the origin. This action has an equivariant momentum map given by $\mathbf{J}(z)=|z|^{2} / 2$ so that $S:=\mathbf{J}^{-1}(0)=0$. Then $D_{0}=\{0\}$ so that $D_{0}^{\circ}=\mathbb{C}$ and, hence, $B\left(D_{0}^{\circ}\right)=\mathbb{C}$, since the Poisson structure on $\mathbb{C}$ is induced by the standard symplectic structure which is nondegenerate. On the other hand, $T_{0} S=0$, so that $T_{0} S+D_{0}=\{0\}$, which contradicts (2.3). However, $\Delta_{0}=\{0\}$ and thus this is consistent with (2.2).

We now study the functoriality property of Poisson reduction, that will be used to reduce the dynamics.

PROPOSITION 2.14. Let $\left(M_{j}, S_{j}, D_{j}\right) j=1,2$, be Poisson reducible. We denote the Poisson bracket of $M_{j}$ by $\{\cdot, \cdot\}_{j}$. Let $\varphi: M_{1} \rightarrow M_{2}$ be a Poisson map such that $\varphi\left(S_{1}\right) \subset S_{2}$, and $T \varphi\left(D_{1}\right) \subset D_{2}$ (therefore $\varphi$ maps the equivalence classes of $\Phi_{1}$ into the equivalence classes of $\Phi_{2}$ ). Then $\varphi$ induces a unique smooth Poisson map $\widehat{\varphi}: S_{1} / \Phi_{1} \rightarrow S_{2} / \Phi_{2}$, characterized by $\pi_{2} \circ \varphi \circ i_{1}=\widehat{\varphi} \circ \pi_{1}$, where $i_{j}: S_{j} \hookrightarrow M_{j}$ are the inclusions and $\pi_{j}: S_{j} \rightarrow S_{j} / \Phi_{j}$ are the projections. We call $\widehat{\varphi}$ the reduction of $\varphi$.

Proof. By the hypotheses on $\varphi$, the map $\widehat{\varphi}$ exists, is smooth, and is unique. We show that it is Poisson. Let $f, g \in C^{\infty}\left(S_{2} / \Phi_{2}\right), m \in S_{1}$, and $F, G \in C^{\infty}\left(M_{2}\right)$ be local extensions at $\varphi(m) \in S_{2}$ of $f \circ \pi_{2}, g \circ \pi_{2} \in C^{\infty}\left(S_{2}\right)$ respectively, such that $\left.\mathbf{d} F\right|_{D_{2}}=\left.\mathbf{d} G\right|_{D_{2}}=0$. Then

$$
\begin{aligned}
\widehat{\varphi}^{*}\{f, g\}_{S_{2} / \Phi_{2}}\left(\pi_{1}(m)\right) & =\{f, g\}_{S_{2} / \Phi_{2}}\left(\left(\widehat{\varphi} \circ \pi_{1}\right)(m)\right) \\
& =\{f, g\}_{S_{2} / \Phi_{2}}\left(\left(\pi_{2} \circ \varphi\right)(m)\right) \\
& =\{F, G\}_{2}(\varphi(m)) .
\end{aligned}
$$

Note that $F \circ \varphi, G \circ \varphi \in C^{\infty}\left(M_{1}\right)$ are smooth local extensions at $m \in S_{1}$ of $f \circ \widehat{\varphi} \circ \pi_{1}, g \circ \widehat{\varphi} \circ \pi_{1} \in C^{\infty}\left(S_{1}\right)$, respectively, which satisfy $\left.\mathbf{d}(F \circ \varphi)\right|_{D_{1}}=$ $\left.\mathbf{d}(G \circ \varphi)\right|_{D_{1}}=0$ by the chain rule and the hypothesis $T \varphi\left(D_{1}\right) \subset D_{2}$. Therefore,

$$
\left\{\widehat{\varphi}^{*} f, \widehat{\varphi}^{*} g\right\}_{S_{1} / \Phi_{1}}\left(\pi_{1}(z)\right)=\{F \circ \varphi, G \circ \varphi\}_{1}(z)=\{F, G\}_{2}(\varphi(z)),
$$

which coincides with (2.4) thereby proving the proposition.

Within the framework of Poisson manifolds, the natural identification between derivations on the ring of smooth real-valued functions and vector fields, allows us to associate to each function on the manifold a Hamiltonian vector field. In the case 
of Poisson varieties like $\left(S / \Phi, C^{\infty}(S / \Phi)\right)$, we need something more general to introduce dynamics since, in general, $S / \Phi$ is not a smooth manifold and, therefore, defining vector fields is not always possible.

DEFINITION 2.15. Let $(M, S, D)$ be a Poisson reducible system and let $h \in C^{\infty}$ $(S / \Phi)$. We define the Hamiltonian flow associated to $h$ as the smooth map $F_{t}^{S / \Phi}$ : $S / \Phi \rightarrow S / \Phi$ such that for any $f \in C^{\infty}(S / \Phi)$ and any $z \in S / \Phi$, we have

$$
\frac{\mathrm{d}}{\mathrm{d} t} f\left(F_{t}^{S / \Phi}(z)\right)=\{f, h\}_{S / \Phi}\left(F_{t}^{S / \Phi}(z)\right)
$$

Note that within this framework there is no standard Existence and Uniqueness Theorem, as is the case for flows associated to Hamiltonian vector fields on smooth manifolds. In fact, these two issues need to be addressed separately. The following result shows that existence is always guaranteed.

THEOREM 2.16 (Reduction of the dynamics). Let $(M, S, D)$ be a Poisson reducible system and let $h \in C^{\infty}(M)$ be a function such that $\left.\mathbf{d} h\right|_{D}=0$ and whose Hamiltonian flow $F_{t}$ preserves the subset $S$, that is, for any time $t, F_{t}(S) \subset S$. Suppose also that for any $t, T F_{t}(D) \subset D$. Then there is a function $h^{S / \Phi} \in C^{\infty}(S / \Phi)$ uniquely defined by the relation $h^{S / \Phi} \circ \pi=h \circ i$, called the reduced Hamiltonian, for which the reduction $F_{t}^{S / \Phi}$ of $F_{t}$ is a Hamiltonian flow induced by $h^{S / \Phi}$. In addition, $F_{t}^{S / \Phi}$ is a Poisson map.

Proof. The condition $\left.\mathbf{d} h\right|_{D}=0$ guarantees that $h$ is constant on the equivalence classes of $\Phi$ and therefore the relation $h^{S / \Phi} \circ \pi=h \circ i$ defines $h^{S / \Phi}$ uniquely. Proposition 2.14 ensures the existence of $F_{t}^{S / \Phi}: S / \Phi \rightarrow S / \Phi$ as the unique Poisson mapping satisfying the equality $\pi \circ F_{t} \circ i=F_{t}^{S / \Phi} \circ \pi$. We verify that $F_{t}^{S / \Phi}$ is a Hamiltonian flow for $h^{S / \Phi}$. Notice that, by construction, $h$ is a smooth extension of $h^{S / \Phi} \circ \pi$. Thus, if $f^{S / \Phi} \in C^{\infty}(S / \Phi)$ is arbitrary, let $f \in C^{\infty}(M)$ be a smooth local extension at $F_{t_{0}}(m) \in S$ of $f^{S / \Phi} \circ \pi$. By the flow property, for small $\left|t-t_{0}\right|, f$ is also a smooth local extension at $F_{t}(m)$ of $f^{S / \Phi} \circ \pi$. Thus, we get for such $t$

$$
\begin{aligned}
\frac{\mathrm{d}}{\mathrm{d} t} & f^{S / \Phi}\left(F_{t}^{S / \Phi}(\pi(m))\right) \\
& =\frac{\mathrm{d}}{\mathrm{d} t} f^{S / \Phi}\left(\left(\pi \circ F_{t} \circ i\right)(m)\right)=\frac{\mathrm{d}}{\mathrm{d} t} f\left(F_{t}(m)\right) \\
& =\{f, h\}\left(F_{t}(m)\right)=\left\{f^{S / \Phi}, h^{S / \Phi}\right\}_{S / \Phi}\left(\pi\left(F_{t}(m)\right)\right) \\
& =\left\{f^{S / \Phi}, h^{S / \Phi}\right\}_{S / \Phi}\left(F_{t}^{S / \Phi}(\pi(m))\right),
\end{aligned}
$$

which proves the claim.

Let us remark again that $F_{t}^{S / \Phi}$ may not be the unique Hamiltonian flow associated to $h^{S / \Phi}$. The following proposition, due to Sjamaar and Lerman [5, 15], describes a situation in which the uniqueness of the reduced flow is guaranteed. 
PROPOSITION 2.17. Let $(M, S, D)$ be a Poisson reducible system. If the functions in $C^{\infty}(S / \Phi)$ separate points, then each Hamiltonian $h^{S / \Phi} \in C^{\infty}(S / \Phi)$ has a unique associated Hamiltonian flow.

Proof. The existence is guaranteed by the previous theorem since the reduction $F_{t}^{S / \Phi}$ of the Hamiltonian flow $F_{t}$ associated to any smooth local extension $h \in$ $C^{\infty}(M)$ of $h^{S / \Phi} \circ \pi$ at an arbitrary point, such that $\left.\mathbf{d} h\right|_{D}=0$, does the job. Suppose now that $G_{t}^{S / \Phi}$ is another Hamiltonian flow for $h^{S / \Phi}$. Since by hypothesis, the functions in $C^{\infty}(S / \Phi)$ separate points, it is enough to show that for any $f^{S / \Phi} \in$ $C^{\infty}(S / \Phi), \pi(m) \in S / \Phi$, and any time $t$,

$$
f^{S / \Phi}\left(G_{t}^{S / \Phi}\left(F_{-t}^{S / \Phi}(\pi(m))\right)\right)=f^{S / \Phi}(\pi(m)) .
$$

This identity holds as a consequence of the following computation, in which we use the chain rule and the fact that $F_{-t}^{S / \Phi}$ is a Hamiltonian flow for $-h^{S / \Phi}$ :

$$
\begin{aligned}
\frac{\mathrm{d}}{\mathrm{d} t} f^{S / \Phi}\left(G_{t}^{S / \Phi}\left(F_{-t}^{S / \Phi}(\pi(m))\right)\right) \\
=\left\{f^{S / \Phi}, h^{S / \Phi}\right\}_{S / \Phi}\left(G_{t}^{S / \Phi}\left(F_{-t}^{S / \Phi}(\pi(m))\right)\right)+ \\
\quad+\left\{f^{S / \Phi} \circ G_{t}^{S / \Phi},-h^{S / \Phi} \circ G_{t}^{S / \Phi}\right\}_{S / \Phi}\left(F_{-t}^{S / \Phi}(\pi(m))\right)=0 .
\end{aligned}
$$

since the flow $G_{t}^{S / \Phi}$ is Poisson by Theorem 2.16.

This result is particularly relevant when the distribution $D$ is given by the proper action of a Lie group, since in this case, the hypothesis on the separation of points always holds.

\section{Singular Poisson, Point, and Orbit Reduction}

We will now use the results just proved as the main tool to study the reduction of Poisson structures by the proper and canonical action of a Lie group. The simplest case is given in the following theorem.

THEOREM 3.1 (Singular Poisson reduction). Let $(M,\{\cdot, \cdot\})$ be a Poisson manifold and let $\Psi: G \times M \rightarrow M$ be a smooth proper canonical action. Then the following hold:

(i) The pair $\left(C^{\infty}(M / G),\{\cdot, \cdot\}_{M / G}\right)$ is a Poisson algebra, where the Poisson bracket $\{\cdot, \cdot\}_{M / G}$ is characterized by $\{f, g\}_{M / G} \circ \pi=\{f \circ \pi, g \circ \pi\}$, for any $f, g \in C^{\infty}(M / G) ; \pi: M \rightarrow M / G$ denotes the canonical smooth projection.

(ii) Let $h$ be a $G$-invariant function on $M$. The Hamiltonian flow $F_{t}$ of $X_{h}$ commutes with the $G$-action, so it induces a flow $F_{t}^{M / G}$ on $M / G$ which is Poisson and is characterized by $\pi \circ F_{t}=F_{t}^{M / G} \circ \pi$.

(iii) The flow $F_{t}^{M / G}$ is the unique Hamiltonian flow defined by the function $[h] \in$ $C^{\infty}(M / G)$ which is given by $[h] \circ \pi=h$. We will call $[h]$ the reduced Hamiltonian. 
Proof. (i) This part can be obtained as a corollary to Theorem 2.12 by taking $M=S$, and $D \subset T M$ the distribution given by $D_{m}=\mathfrak{g} \cdot m$. This distribution is smooth since for every $m \in M$, if $\left\{\xi^{1}, \ldots, \xi^{n}\right\}$ is a basis of the Lie algebra $\mathfrak{g}$, the evaluation of the vector fields $\xi_{M}^{1}, \ldots, \xi_{M}^{n}$ at $m$, spans $D_{m}$. The distribution $D$ is also trivially integrable since, by construction, the orbit $G \cdot m$ is a submanifold of $M$ such that $D_{m}=T_{m}(G \cdot m)=\mathfrak{g} \cdot m$, for arbitrary $m \in M$. The canonical character of the $G$-action guarantees that $D$ is Poisson in the sense of Definition 2.8. Remark that the distribution $D$ satisfies trivially the extension property, as well as the hypothesis of Theorem 2.12 since $B\left(\Delta_{m}\right) \subset T_{m} M \subset T_{m} M+\left[\Delta_{M}^{S}\right]^{\circ}$. This guarantees that $\left(C^{\infty}(M / G),\{\cdot, \cdot\}_{M / G}\right)$ is a Poisson algebra.

(ii) Since the Lie group $G$ acts canonically on $M$ and the Hamiltonian $h$ is $G$ invariant, the Hamiltonian flow associated to $h$ satisfies that $\Psi_{g} \circ F_{t}=F_{t} \circ \Psi_{g}$ for any $g \in G$ and therefore, for any $\xi \in \mathfrak{g}$, any $m \in M$, and any time $t$

$$
\begin{aligned}
T_{m} F_{t} \cdot \xi_{M}(m) & =\left.\frac{\mathrm{d}}{\mathrm{d} s}\right|_{s=0} F_{t}(\exp s \xi \cdot m) \\
& =\left.\frac{\mathrm{d}}{\mathrm{d} s}\right|_{s=0} \exp s \xi \cdot F_{t}(m)=\xi_{M}\left(F_{t}(m)\right),
\end{aligned}
$$

which implies that $T F_{t}(D) \subset D$. The claim follows from Proposition 2.14.

(iii) is a corollary of Theorem 2.16. The uniqueness follows from Proposition 2.17, and the properness of the action.

At this point we will assume that $M$ is not only Poisson, but also symplectic, and that the canonical action of $G$ on $M$ is proper and has an associated globally equivariant momentum map $\mathbf{J}: M \rightarrow \mathfrak{g}^{*}$, that is, the action is globally Hamiltonian. The natural step to take in this situation is studying point and orbit reduction. Regarding the former, recall that in the regular case [13], if $M$ was a symplectic manifold, so was the point reduced space $M_{\mu}:=\mathbf{J}^{-1}(\mu) / G_{\mu}$, where $G_{\mu}$ denotes the coadjoint isotropy subgroup of $\mu \in \mathfrak{g}^{*}$. If we are in a genuinely, singular situation, the space $\mathbf{J}^{-1}(\mu) / G_{\mu}$ is not even a manifold; however, it can be shown that in the sense of Definition 2.11, it is endowed with a Poisson structure. The construction of this Poisson structure constitutes the Universal Reduction Procedure of Arms, Cushman and Gotay [3] which is described in detail in the following theorem.

THEOREM 3.2 (Singular point reduction). Let $(M, \omega)$ be a symplectic manifold and let $G$ be a Lie group acting properly on $M$ in a globally Hamiltonian fashion with associated equivariant momentum map $\mathbf{J}: M \rightarrow \mathfrak{g}^{*}$. Let $\mu \in \mathfrak{g}^{*}$ be a value of $\mathbf{J}$ and denote by $G_{\mu}$ the isotropy of $\mu$ under the coadjoint action of $G$ on $\mathfrak{g}^{*}$. The following hold:

(i) The set $M_{\mu}:=\mathbf{J}^{-1}(\mu) / G_{\mu}$ is such that the pair $\left(C^{\infty}\left(M_{\mu}\right),\{\cdot, \cdot\}_{M_{\mu}}\right)$ is a Poisson algebra, with Poisson bracket $\{\cdot, \cdot\}_{M_{\mu}}$ characterized by

$$
\left\{f_{\mu}, g_{\mu}\right\}_{M_{\mu}}\left([m]_{\mu}\right)=\{f, g\}(m),
$$


for any $f_{\mu}, g_{\mu} \in C^{\infty}\left(M_{\mu}\right)$. The functions $f, g \in C^{\infty}(M)^{G}$ are arbitrary smooth local extensions at $m \in \mathbf{J}^{-1}(\mu)$ of $f_{\mu} \circ \pi_{\mu}, g_{\mu} \circ \pi_{\mu} \in C^{\infty}\left(\mathbf{J}^{-1}(\mu)\right)^{G_{\mu}}$, where $\pi_{\mu}: \mathbf{J}^{-1}(\mu) \rightarrow M_{\mu}$ is the canonical smooth projection, and $[\mathrm{m}]_{\mu}:=$ $\pi_{\mu}(m) \in M_{\mu}$.

(ii) Let $h \in C^{\infty}(M)^{G}$ be a $G$-invariant Hamiltonian. The Hamiltonian flow $F_{t}$ of $h$ leaves the connected components of $\mathbf{J}^{-1}(\mu)$ invariant and commutes with the $G$-action, so it induces a Poisson flow $F_{t}^{\mu}$ on $M_{\mu}$, uniquely determined by

$$
\pi_{\mu} \circ F_{t} \circ i_{\mu}=F_{t}^{\mu} \circ \pi_{\mu},
$$

where $i_{\mu}: \mathbf{J}^{-1}(\mu) \hookrightarrow M$ is the canonical injection.

(iii) The flow $F_{t}^{\mu}$ is the unique Hamiltonian flow in $\left(M_{\mu},\{\cdot, \cdot\}_{M_{\mu}}\right)$, with Hamiltonian function $h_{\mu} \in C^{\infty}\left(M_{\mu}\right)$ defined by $h_{\mu} \circ \pi_{\mu}=h \circ i_{\mu}$. We will call $h_{\mu}$ the reduced Hamiltonian.

(iv) Let $k \in C^{\infty}(M)^{G}$ be another $G$-invariant function. Then, $\{h, k\}$ is also $G$ invariant and $\{h, k\}_{\mu}=\left\{h_{\mu}, k_{\mu}\right\}_{M_{\mu}}$.

Proof. Once more, we will obtain this result as a corollary to Theorem 2.12 taking $M$ as the Poisson manifold, $\mathbf{J}^{-1}(\mu)$ as the stratified subset $S$, and $D$ as the distribution given by the tangent spaces to the $G$-orbits in $\mathbf{J}^{-1}(\mu)$, that is, for any $m \in \mathbf{J}^{-1}(\mu), D_{m}=\mathfrak{g} \cdot m$. We verify that $\mathbf{J}^{-1}(\mu)$ is a stratified subset in the sense of Definition 2.4 and that $D$ is a smooth, integrable, Poisson distribution, adapted to the stratification of $\mathbf{J}^{-1}(\mu)$, for which the extension property holds.

Firstly, the equivariance of $\mathbf{J}$ with respect to the $G$-action implies that there is a well-defined continuous $G_{\mu}$-action on the topological space $\mathbf{J}^{-1}(\mu)$. Since the subset $\mathbf{J}^{-1}(\mu)$ and the subgroup $G_{\mu}$ are closed in $M$ and $G$, respectively, the $G_{\mu}$ action on $\mathbf{J}^{-1}(\mu)$ is proper and therefore a standard result (see, for instance [6-8]) guarantees that $\mathbf{J}^{-1}(\mu)$ can be stratified using the orbit type manifolds associated to the $G_{\mu}$-action, that is, $\mathbf{J}^{-1}(\mu)$ is a stratified subset of $M$ with strata the submanifolds of $M$

$$
\left(\mathbf{J}^{-1}(\mu)\right)_{(H)}^{G_{\mu}}:=\mathbf{J}^{-1}(\mu) \cap M_{(H)}^{G_{\mu}},
$$

for any isotropy subgroup $H \subset G_{\mu}$. Recall that

$$
M_{(H)}^{G_{\mu}}:=\left\{z \in M \mid G_{z} \text { is conjugate to } H \text { in } G_{\mu}\right\} .
$$

By the Bifurcation Lemma (see [4, 14]), for any $m \in M$, range $\left(T_{m} \mathbf{J}\right)=\left(\mathfrak{g}_{m}\right)^{\circ}$, where $\mathfrak{g}_{m}$ is the Lie algebra of the isotropy subgroup $G_{m}$, and $\left(\mathfrak{g}_{m}\right)^{\circ}:=\{\mu \in$ $\left.\mathfrak{g}^{*}|\mu| \mathfrak{g}_{m}=0\right\}$ denotes the annihilator in $\mathfrak{g}^{*}$ of $\mathfrak{g}_{m}$. Note that this proves that $\left.\mathbf{J}\right|_{M_{(H)}^{G \mu}}$ is a constant rank map and, hence, by the Subimmersion Theorem (see [2, Theorem 3.5.17]),

$$
\left(\left.\mathbf{J}\right|_{M_{(H)}^{G_{\mu}}}\right)^{-1}(\mu)=\mathbf{J}^{-1}(\mu) \cap M_{(H)}^{G_{\mu}}=\left(\mathbf{J}^{-1}(\mu)\right)_{(H)}^{G_{\mu}}
$$

is a submanifold of $M_{(H)}^{G_{\mu}}$ and therefore of $M$. 
Secondly, the distribution $D$ is smooth since it is induced by a smooth group action. We now verify that it is adapted to the stratification of $\mathbf{J}^{-1}(\mu)$ by $G_{\mu}$-orbit types. Recall that the Subimmersion Theorem states that for any $m \in\left(\mathbf{J}^{-1}(\mu)\right)_{(H)}^{G_{\mu}}$,

$$
\begin{aligned}
T_{m}\left[\left(\mathbf{J}^{-1}(\mu)\right)_{(H)}^{G_{\mu}}\right] & =T_{m}\left(\left(\left.\mathbf{J}\right|_{M_{(H)}^{G_{\mu}}}\right)^{-1}(\mu)\right)=\left.\operatorname{ker} T_{m} \mathbf{J}\right|_{M_{(H)}^{G_{\mu}}} \\
& =\operatorname{ker} T_{m} \mathbf{J} \cap T_{m} M_{(H)}^{G_{\mu}},
\end{aligned}
$$

and therefore, using the Reduction Lemma and the $G_{\mu}$-invariance of $M_{(H)}^{G_{\mu}}$,

$$
\begin{aligned}
D_{m} \cap T_{m}\left[\left(\mathbf{J}^{-1}(\mu)\right)_{(H)}^{G_{\mu}}\right] & =\operatorname{ker} T_{m} \mathbf{J} \cap T_{m} M_{(H)}^{G_{\mu}} \cap \mathfrak{g} \cdot m \\
& =\mathfrak{g}_{\mu} \cdot m \cap T_{m} M_{(H)}^{G_{\mu}}=\mathfrak{g}_{\mu} \cdot m .
\end{aligned}
$$

This implies that $D$ coincides, stratum by stratum, with the smooth integrable distribution induced by the $G_{\mu}$-action, which guarantees that $D$ is integrable and adapted to the stratified subset $\mathbf{J}^{-1}(\mu)$. As in Theorem 3.1, the canonical character of the $G$-action implies that the distribution $D$ is Poisson. The extension property of $D$ follows from the following proposition whose proof follows in a straightforward manner from the use of normal forms (see [14]).

PROPOSITION 3.3. Let $(M, \omega)$ be a symplectic manifold and let $G$ be a Lie group acting properly on $M$ in a globally Hamiltonian fashion with associated equivariant momentum map $\mathbf{J}: M \rightarrow \mathfrak{g}^{*}$. Let $m \in M$ and denote $\mathbf{J}(m)=\mu, H:=G_{m}$. Then every $f \in C^{\infty}\left(\mathbf{J}^{-1}(\mu)\right)^{G_{\mu}}$ (respectively, $\left.f \in C^{\infty}\left(\mathbf{J}^{-1}(\mu) \cap M_{(H)}^{G_{\mu}}\right)^{G_{\mu}}\right)$ admits a local $G$-invariant extension at $m$ to $C^{\infty}(M)^{G}$.

Finally, in order to show that $M_{\mu}$ is Poisson, we use Theorem 2.12 to prove that the triplet $\left(M, \mathbf{J}^{-1}(\mu), D\right)$ is Poisson reducible, that is, we will verify for arbitrary $m \in \mathbf{J}^{-1}(\mu)$ that

$$
B\left(\Delta_{m}\right) \subset T_{m}\left(\mathbf{J}^{-1}(\mu)\right)+\left[\Delta_{m}^{\mathbf{J}^{-1}(\mu)}\right]^{\circ} .
$$

Indeed, if $F \in C^{\infty}(M)^{G_{\mu}}$ and $H=G_{m}$, we will show that

$$
\begin{aligned}
X_{F}(m) & \in T_{m}\left[\left(\mathbf{J}^{-1}(\mu)\right)_{(H)}^{G_{\mu}}\right]+\left[\Delta_{m}^{\mathbf{J}^{-1}(\mu)}\right]^{\circ} \\
& =\left[\operatorname{ker} T_{m} \mathbf{J} \cap T_{m} M_{(H)}^{G_{\mu}}\right]+\left[\Delta_{m}^{\mathbf{J}^{-1}(\mu)}\right]^{\circ} .
\end{aligned}
$$

To see this, let

$$
\begin{aligned}
\alpha_{m} & \in\left[\left[\operatorname{ker} T_{m} \mathbf{J} \cap T_{m} M_{(H)}^{G_{\mu}}\right]+\left[\Delta_{m}^{\mathbf{J}^{-1}(\mu)}\right]^{\circ}\right]^{\circ} \\
& =\left[\operatorname{ker} T_{m} \mathbf{J} \cap T_{m} M_{(H)}^{G_{\mu}}\right]^{\circ} \cap \Delta_{m}^{\mathbf{J}^{-1}(\mu)},
\end{aligned}
$$

so that $\alpha_{m}=\mathbf{d} K(m)$ for some $K \in C^{\infty}(M)^{G}$, constant on $U_{m} \cap \mathbf{J}^{-1}(\mu)$, where $U_{m}$ is an open neighborhood of $m$ in $M$. Then,

$$
\left\langle\alpha_{m}, X_{F}(m)\right\rangle=\{K, F\}(m)=X_{F}[K](m) .
$$


However, by Noether's Theorem, the Hamiltonian flow $F_{t}$ of $X_{F}$ preserves the level sets of $\mathbf{J}$, in particular $\mathbf{J}^{-1}(\mu)$. Therefore,

$$
X_{F}[K](m)=\left.\frac{\mathrm{d}}{\mathrm{d} t}\right|_{t=0} K\left(F_{t}(m)\right)=0,
$$

since $\left.K\right|_{\mathbf{J}^{-1}(\mu)}=0$. This proves the required condition on $X_{F}(m)$ and, hence, implies that $\left(M_{\mu}, C^{\infty}\left(M_{\mu}\right)\right)$ is a Poisson algebra with bracket $\{\cdot, \cdot\}_{M_{\mu}}$ defined by

$$
\left\{f_{\mu}, g_{\mu}\right\}_{M_{\mu}}\left([m]_{\mu}\right)=\{f, g\}(m),
$$

for any $f_{\mu}, g_{\mu} \in C^{\infty}\left(M_{\mu}\right)$, and $f, g \in C^{\infty}(M)^{G}$ arbitrary smooth local $G$-invariant extensions at $m$ of $f_{\mu} \circ \pi_{\mu}, g_{\mu} \circ \pi_{\mu} \in C^{\infty}\left(\mathbf{J}^{-1}(\mu)\right)^{G_{\mu}}$, whose existence is again guaranteed by Proposition 3.3.

The remaining points are a straightforward consequences of Noether's Theorem, Proposition 2.14, and Theorem 2.16. The uniqueness of the flow for the reduced Hamiltonian follows from Proposition 2.17, and the properness of the action.

A theorem completely identical can be stated for the singular orbit reduced space $M_{\mathcal{O}_{\mu}}:=\mathbf{J}^{-1}\left(\mathcal{O}_{\mu}\right) / G$. The only difference in the proof, with respect to the one corresponding to Theorem 3.2, is that in this case $\mathbf{J}^{-1}\left(\mathcal{O}_{\mu}\right)$ will play the role of $S$, which will be stratified by means of the orbit types corresponding to the $G$-action defined on it. The extension property follows directly in this case from Proposition 2.7, due to the $G$-invariance of $\mathbf{J}^{-1}\left(\mathcal{O}_{\mu}\right)$.

THEOREM 3.4 (Singular orbit reduction). Let $(M, \omega)$ be a symplectic manifold and let $G$ be a Lie group acting properly on $M$ in a globally Hamiltonian fashion with associated equivariant momentum map $\mathbf{J}: M \rightarrow \mathfrak{g}^{*}$. Let $\mu \in \mathfrak{g}^{*}$ be a value of $\mathbf{J}$, and denote by $\mathcal{O}_{\mu}$ the orbit of $\mu$ under the coadjoint action of $G$ on $\mathfrak{g}^{*}$. Then the following hold:

(i) The set $M_{\mathcal{O}_{\mu}}:=\mathbf{J}^{-1}\left(\mathcal{O}_{\mu}\right) / G$ is such that the pair $\left(C^{\infty}\left(M_{\mathcal{O}_{\mu}}\right),\{\cdot, \cdot\}_{M_{\mathcal{O}_{\mu}}}\right)$ is a Poisson algebra, with Poisson bracket $\{\cdot, \cdot\}_{M_{\mathcal{O}_{\mu}}}$, characterized by

$$
\left\{f_{\mathcal{O}_{\mu}}, g_{\mathcal{O}_{\mu}}\right\}_{M_{\mathcal{O}_{\mu}}}\left([m]_{\mathcal{O}_{\mu}}\right)=\{f, g\}(m),
$$

for any $f_{\mathcal{O}_{\mu}}, g_{\mathcal{O}_{\mu}} \in C^{\infty}\left(M_{\mathcal{O}_{\mu}}\right)$. The functions $f, g \in C^{\infty}(M)^{G}$ are arbitrary local extensions at $m$ of $f_{\mathcal{O}_{\mu}} \circ \pi_{\mathcal{O}_{\mu}}, g_{\mathcal{O}_{\mu}} \circ \pi_{\mathcal{O}_{\mu}} \in C^{\infty}\left(\mathbf{J}^{-1}\left(\mathcal{O}_{\mu}\right)\right)^{G}$, where $\pi_{\mathcal{O}_{\mu}}: \mathbf{J}^{-1}(\mu) \rightarrow M_{\mathcal{O}_{\mu}}$ is the canonical smooth projection and $[m]_{\mathcal{O}_{\mu}}:=$ $\pi_{\mathcal{O}_{\mu}}(m) \in M_{\mathcal{O}_{\mu}}$.

(ii) Let $h \in C^{\infty}(M)^{G}$ be a $G$-invariant Hamiltonian. The Hamiltonian flow $F_{t}$ of $h$ leaves the connected components of $\mathbf{J}^{-1}\left(\mathcal{O}_{\mu}\right)$ invariant and commutes with the $G$-action, so it induces a flow $F_{t}^{\mathcal{O}_{\mu}}$ on $M_{\mathcal{O}_{\mu}}$, uniquely determined by

$$
\pi_{\mathcal{O}_{\mu}} \circ F_{t} \circ i_{\mathcal{O}_{\mu}}=F_{t}^{\mathcal{O}_{\mu}} \circ \pi_{\mathcal{O}_{\mu}},
$$

where $i_{\mathcal{O}_{\mu}}: \mathbf{J}^{-1}\left(\mathcal{O}_{\mu}\right) \hookrightarrow M$ is the canonical injection. 
(iii) The flow $F_{t}^{\mathcal{O}_{\mu}}$ is the unique Hamiltonian flow in $\left(M_{\mathcal{O}_{\mu}},\{\cdot, \cdot\}_{M_{\mathcal{O}_{\mu}}}\right)$, with Hamiltonian function $h_{\mathcal{O}_{\mu}} \in C^{\infty}\left(M_{\mathcal{O}_{\mu}}\right)$ defined by $h_{\mathcal{O}_{\mu}} \circ \pi_{\mathcal{O}_{\mu}}=h \circ i_{\mathcal{O}_{\mu}}$. We will call $h_{\mathcal{O}_{\mu}}$ the reduced Hamiltonian.

(iv) Let $k \in C^{\infty}(M)^{G}$ be another $G$-invariant function. Then, $\{h, k\}$ is also $G$ invariant and $\{h, k\}_{\mathcal{O}_{\mu}}=\left\{h_{\mathcal{O}_{\mu}}, k_{\mathcal{O}_{\mu}}\right\}_{M_{\mathcal{O}_{\mu}}}$.

Remark 3.5. The Poisson algebras $\left(C^{\infty}\left(M_{\mu}\right),\{\cdot, \cdot\}_{M_{\mu}}\right)$ and $\left(C^{\infty}\left(M_{\mathcal{O}_{\mu}}\right)\right.$, $\left.\{\cdot, \cdot\}_{\mathcal{O}_{\mu}}\right)$ are in general degenerate and, consequently, have nontrivial symplectic leaves. It can be shown (see [14]) that these leaves correspond to the singular symplectic reduced spaces of Sjamaar and Lerman [15], and Bates and Lerman [5].

\section{References}

1. Abraham, R. and Marsden, J. E.: Foundations of Mechanics, 2nd edn, Addison-Wesley, New York, 1978.

2. Abraham, R., Marsden, J. E., and Ratiu, T. S.: Manifolds, Tensor Analysis, and Applications, Appl. Math. Sci., Springer-Verlag, New York, 1988.

3. Arms J. M., Cushman R., and Gotay M. J.: A universal reduction procedure for Hamiltonian group actions, in: T. S. Ratiu (ed.), The Geometry of Hamiltonian Systems, Springer-Verlag, New York, 1991, pp. 33-51.

4. Arms, J. M., Marsden, J. E., and Moncrief, V.: Symmetry and bifurcations of momentum mappings, Comm. Math. Phys. 78 (1975), 455-478.

5. Bates, L. and Lerman, E.: Proper group actions and symplectic stratified spaces, Pacific J. Math. 181(2) (1997), 201-229.

6. Bourguignon, J. P.: Une stratification de l'espace des structures riemanniennes, Compositio Math. 30 (1975), 1-41.

7. Bredon, G. E.: Introduction to Compact Transformation Groups, Academic Press, New York, 1972.

8. Burghelea, D., Albu, A., and Ratiu, T. S.: Compact Lie Group Actions (in Romanian), Monografii Matematice 5, Universitatea Timisoara, 1975.

9. Cartan, É.: Leçons sur les invariants intégraux, Hermann, Paris, 1922.

10. Libermann, P. and Marle, C.-M.: Symplectic Geometry and Analytical Mechanics, D. Reidel, Dordrecht, 1987.

11. Marsden, J. E. and Ratiu, T. S.: Reduction of Poisson manifolds, Lett. Math. Phys. 11 (1986), 161-169.

12. Marsden, J. E. and Ratiu, T. S.: Introduction to Mechanics and Symmetry, Texts Appl. Math. 17, Springer-Verlag, New York, 1994.

13. Marsden, J. E. and Weinstein, A.: Reduction of symplectic manifolds with symmetry, Rep. Math. Phys. 5(1) (1974), 121-130.

14. Ortega, J.-P. and Ratiu, T. S.: Symmetry, Reduction, and Stability in Hamiltonian Systems, in preparation.

15. Sjamaar, R. and Lerman, E.: Stratified symplectic spaces and reduction, Ann. of Math. 134 (1991), 375-422.

16. Stefan, P.: Accessible sets, orbits and foliations with singularities, Proc. London Math. Soc. 29 (1974), 699-713.

17. Sussman, H.: Orbits of families of vector fields and integrability of distributions, Trans. Amer. Math. Soc. 180 (1973), 171-188. 